\title{
Fabrication and characterization of novel tentacle-type adsorbent for resolution of chiral drugs
}

\author{
ZHU WeiXia, WANG QuanYi, DU KaiFeng, YAO Shun \& SONG Hang* \\ Department of Pharmaceutical \& Biological Engineering, School of Chemical Engineering, Sichuan University, Chengdu 610065, China
}

Received December 14, 2012; accepted February 21, 2013; published online June 21, 2013

\begin{abstract}
Novel tentacle-type chiral adsorbent based on silica gels modified with $\beta$-cyclodextrin $(\beta$-CD) and polyvinyl alcohol(PVA) is synthesized by surface grafting technique. The adsorption behavior of chiral pharmaceuticals using the resulting adsorbents is investigated. In the study, FT-IR and thermogravimetric analysis (TGA) are employed to characterize the chemical and physical properties of the adsorbent; mandelic acid (MA) serves as model solute to evaluate the sorption capacity and selectivity of the adsorbent. The experimental results show that the novel tentacle-type adsorbent is possessed of larger equilibrium adsorption capacity and better selectivity over the conventional one and could be as potential chiral stationary phase (CSPs).
\end{abstract}

tentacle-type adsorbent, adsorptive selectivity, chiral resolution, MA

Citation: Zhu W X, Wang Q Y, Du K F, et al. Fabrication and characterization of novel tentacle-type adsorbent for resolution of chiral drugs. Chin Sci Bull, 2013, 58: 3390-3397, doi: 10.1007/s11434-013-5782-6

Chirality is a fundamental feature of many pharmaceutical molecules, which plays an important role for disease treatment. Because of possessing many advantages such as high efficiency, low toxic side effect, small dosage, and so on, chiral pharmaceuticals have provoked great interest and become the orientation of future drug development in recent years [1]. However, enantiomers of chiral pharmaceuticals have shown different pharmacological activity or toxicity [2]. Therefore, separation of chiral pharmaceuticals has become an extremely important and challenging unit operation in the pharmaceutical industry. Up to now, there are so many techniques employed to separate the chiral drugs, such as diastereomer crystallization [3], membrane separation [4], chromatographic chiral separation [5,6] and so on. Among all of these techniques, liquid chromatography (LC) packed with chiral stationary phase (CSPs) is very attractive because it possesses excellent enantioselectivities in a wide range of enantiomers. For this technique, the CSPs are the central unit that relates strongly to the chromatographic performance [7-9]. At present, increasing research efforts have been focused on the fabrication of the novel adsorbent

*Corresponding author (email: hangsong@ @scu.edu.cn) possessing of better enantioselectivity, stability and capacity and their potential application in industry.

For chromatography of CSPs, chiral ligand serves as one of the most important factors that impacts on the chiral resolution. Cyclodextrin (CD), that is a chiral polymer, has been widely used for the separation of enantiomers and other isomers owing to its special ring structure $[10,11]$. However, conventional chiral adsorbent is modified with one layer of chiral ligands on the surface of the carrier, which results in a monolayer adsorption of chiral drugs in the resolution process $[12,13]$. Due to the density limit of ligands, it usually results in the insufficient resolution capacity, and is not suitable for preparative scale resolution of chiral pharmaceuticals [14]. To resolve the problem, a surface grafting technique has been developed to increase the density of ligands in many adsorption materials recently [15-17]. For instance, the long polymer chains with multiple functional groups were grafted onto the pore surface of the materials to form the tentacle-type adsorbents, which was expected that the multi-layer adsorption for the target from the surface to three-dimensional space would exhibit high resolution capacity. The tentacle-type modification has been successfully applied to the adsorption of the metal ions 
[18] and protein separation [19], and the results showed that the adsorption performance has been improved remarkably, especially for separation capacity.

The chiral pharmaceutical molecules possess smaller size, better stability, and lower mass transfer resistance in the absorption process over the biological macromolecules. Based on the above-mentioned features, tentacle-type chiral adsorbent would be more adequate for the chiral pharmaceuticals resolution. Moreover, the flexible structure of the long-chain polymer can reduce effectively the steric hindrance during the adsorption process, which helps to the target adsorption onto the chiral adsorption sites. In addition, polyvinyl alcohol (PVA) is well known for its processability, strength and long-term temperature and $\mathrm{pH}$ stability [20]. Unfortunately, this tentacle-type chiral adsorbent has been scarcely any applied to separate chiral drugs. Inspirited by these, to obtain high-capacity of chiral drugs, a novel tentacle-type adsorbent based on the pore surface of silica gel particles grafted with PVA macromolecules and $\beta$ cyclodextrin $(\beta$-CD) were prepared, which was named $\beta$ CD-PVA-SiO 2 . This novel tentacle-type adsorbent was expected to possess better enantioselectivity, stability and capacity due to the multiple functional groups on PVA. To highlight the novel adsorbents $\left(\beta\right.$-CD-PVA-SiO $\left.{ }_{2}\right)$, the traditional ones $\left(\beta-\mathrm{CD}-\mathrm{SiO}_{2}\right)$ were also prepared in this study. The ligand densities on two types of chiral adsorbents were determined by infrared spectrum and thermogravimetric analysis. And adsorption properties of the chiral adsorbents were investigated using mandelic acid as model solute.

\section{Materials and methods}

\subsection{Chemicals}

Silica gel (particle size, $5 \mu \mathrm{m}$ ) was purchased from the Ocean Chemical Co., Ltd. (Qingdao, China). $\gamma$-Aminopropyltrimethoxysilane and $\gamma$-glycidoxypropyltrimethoxysilane were provided by the Yongchang Chemical Company, Ltd. (Nanjing, China). $\beta$-Cyclodextrin $(\beta$-CD) and polyvinyl alcohol (PVA, molecular weight, $79 \mathrm{kDa}$ ) were obtained from Changzheng Chemical Co., Ltd. (Chengdu, China). The racemic mandelic acid (MA) and its enantiomers (R-MA and S-MA) were produced by Sigma (USA). $n$-Hexane and dehydrated alcohol (HPLC grade) were purchased from Fisher (Loughborough, UK). Other reagents were all of analytical grade received from local sources.

\subsection{Preparation of silica gel functionalized with PVA}

The silica gel functionalized with PVA $\left(\mathrm{PVA}-\mathrm{SiO}_{2}\right)$ was synthesized as follows. Firstly, silica gel activated with hydrochloric acid [21], was soaked in the mixture of dry toluene and $\gamma$-aminopropyltrimethoxysilane. The suspension was refluxed with gentle stirring in an argon atmosphere for $24 \mathrm{~h}$. After filtered and washed with dry toluene and acetone, the aminopropyl silica gel was obtained. Then, $5 \mathrm{~g}$ of aminopropyl silica gel was added to $50 \mathrm{~mL}$ of $0.02 \mathrm{~mol} \mathrm{~L}^{-1}$ glutaraldehyde solution containing $0.01 \mathrm{~mol} \mathrm{~L}^{-1}$ phosphate buffer solution ( $\mathrm{pH}$ 6.2). After reacting for $4 \mathrm{~h}$ at room temperature, the intermediate was washed to neutral solution by water. Subsequently, it was dispersed in the $100 \mathrm{~mL}$ of solution containing $90 \mathrm{~mL}$ of PVA aqueous solution $(5 \%$, $\mathrm{w} / \mathrm{w})$ and $10 \mathrm{~mL}$ of hydrochloric acid $\left(5 \mathrm{~mol} \mathrm{~L}^{-1}\right)$ and reacted for $3 \mathrm{~h}$, and then PVA was grafted onto the silica gel surface through coupling. Finally, the product $\mathrm{PVA}-\mathrm{SiO}_{2}$ was washed by water and dried under vacuum at $80^{\circ} \mathrm{C}$.

\subsection{Synthesis of tentacle-type chiral adsorbent}

In the procedure, $5 \mathrm{~g}$ of $\beta$-CD was firstly dissolved in $100 \mathrm{~mL}$ of dry DMF, and reacted with $0.4 \mathrm{~g}$ of $\mathrm{NaH}$ for $2 \mathrm{~h}$ at room temperature. After filtered, the resultant reacted with $2 \mathrm{~mL}$ of $\gamma$-glycidoxypropyltrimethoxysilane in an argon atmosphere for $5 \mathrm{~h}$ at $90^{\circ} \mathrm{C}$, and further reacted with $4.0 \mathrm{~g}$ of $\mathrm{PVA}-\mathrm{SiO}_{2}$ by refluxing for $24 \mathrm{~h}$ at $120^{\circ} \mathrm{C}$. After the reaction, the tentacle-type chiral adsorbent $\left(\beta\right.$-CD-PVA-SiO $\left.{ }_{2}\right)$ was obtained. The final products was washed successively with $\mathrm{DMF}$, methanol, water, and acetone, and then dried in vacuum at $80^{\circ} \mathrm{C}$.

As a reference, the non-grafted adsorbent was prepared by a similar procedure. Firstly, $5 \mathrm{~g} \beta$-CD was dissolved in $100 \mathrm{~mL}$ dry DMF, and reacted with $0.4 \mathrm{~g} \mathrm{NaH}$ for $2 \mathrm{~h}$ at room temperature. Then the products were filtered and reacted with $2 \mathrm{~mL} \gamma$-glycidoxypropyltrimethoxysilane in an argon atmosphere for $5 \mathrm{~h}$ at $90^{\circ} \mathrm{C}$. Finally, the results mixed with $4.0 \mathrm{~g}$ silica gel which was activated with hydrochloric acid for $24 \mathrm{~h}$ at $120^{\circ} \mathrm{C}$, and the non-grafted adsorbent $\left(\beta-\mathrm{CD}-\mathrm{SiO}_{2}\right)$ were obtained.

\subsection{Characterization and evaluation of the chiral adsorbents}

The adsorbents were characterized by FT-IR obtained using $\mathrm{KBr}$ (Spectrum One, Perkin-Elmer, USA) and Thermogravimetric analysis (TGA) performed on a TA instrument (TG 209F1, Netzsch, Germany), at a heating rate of $10^{\circ} \mathrm{C}$ $\min ^{-1}$ and an air flow of $100 \mathrm{~mL} \mathrm{~min}^{-1}$.

To highlight the tentacle-type chiral adsorbent, MA as model solute was applied to perform the static and dynamic adsorption experiments. Enantiomers of MA in different samples were analyzed on a programmable HPLC (CLASSVP, LC-20AT, $15 \mu \mathrm{L}$ injection loop, SPD-M20A detector, Shimadzu, Japan) packed with a chiral column OD-H (250 $\mathrm{mm} \times 4.6 \mathrm{~mm}$ i.d.) at $254 \mathrm{~nm}, 25^{\circ} \mathrm{C}$ and a flow rate of 0.8 $\mathrm{mL} \min ^{-1}$. The mobile phase consisted of the mixture of $n$-hexane and dehydrated alcohol $(92: 8, \mathrm{v} / \mathrm{v})$ containing $0.5 \%$ trifluoroacetic acid. Samples and mobile phases were filtered through a $0.45 \mu \mathrm{m}$ filter membrane before injection. The software of origin 7.5 was utilized to determine the figures and the relevant parameters. 


\subsection{Static adsorption of MA on two types of adsorbents}

(1) Measurement of kinetic adsorption curve. Briefly as follows, $0.2 \mathrm{~g}$ of absorbent was added into a conical flask containing $100 \mathrm{~mL}$ of aqueous R-MA or S-MA solution with an initial concentration $\left(C_{0}\right)$ of $0.8 \mathrm{mg} \mathrm{mL}^{-1}$. The suspension was incubated at the temperature of 20,30 and $40^{\circ} \mathrm{C}$, and stirred by a magnetic stirrer. Every interval, $1 \mathrm{~mL}$ of sample was periodically collected and centrifuged instantly to determine the MA concentration $\left(C_{\mathrm{t}}, \mathrm{mg} \mathrm{mL}^{-1}\right)$ in supernatant by using the HPLC technique, and then the sample was returned to the flask immediately. The adsorption amounts $\left(Q, \mathrm{mg} \mathrm{g}^{-1}\right)$ of the MA on the adsorbents were calculated according to the eq. (1).

$$
Q=\frac{V \times\left(C_{0}-C_{\mathrm{t}}\right)}{m},
$$

where $V(\mathrm{~mL})$ and $m(\mathrm{~g})$ stand for the solution volume and the weight of the absorbent, respectively.

(2) Measurement of adsorption isotherm with static method. In the procedure, $0.2 \mathrm{~g}$ of absorbent was placed into labeled conical flasks and mixed with $30 \mathrm{~mL}$ of R-MA aqueous solution of different concentrations $\left(C_{0}\right)(0-0.8 \mathrm{mg}$ $\mathrm{mL}^{-1}$ ). Then the suspension was stirred by a magnetic stirrer in an incubator at the temperature of 20,30 and $40^{\circ} \mathrm{C}$ for $24 \mathrm{~h}$ to ensure equilibrium. Finally, the suspensions were centrifuged and the R-MA concentrations $\left(C_{\mathrm{e}}\right)$ in supernatant were detected. The equilibrium adsorption capacity $\left(Q_{\mathrm{e}}\right.$, $\mathrm{mg} \mathrm{g}^{-1}$ ) of R-MA was calculated by the eq. (2).

$$
Q_{\mathrm{e}}=\frac{V\left(C_{0}-C_{\mathrm{e}}\right)}{m},
$$

where $C_{0}$ and $C_{\mathrm{e}}\left(\mathrm{mg} \mathrm{mL}^{-1}\right)$ stand for the initial and equilibrium concentration of R-MA, respectively. $V(\mathrm{~mL})$ and $m$ (g) stand for the solution volume and the weight of the absorbent, respectively. The Langmuir isotherm was used to fit the equilibrium data by using the following equation:

$$
Q_{\mathrm{e}}=\frac{Q_{\mathrm{m}} C_{\mathrm{e}}}{K_{\mathrm{d}}+C_{\mathrm{e}}},
$$

where $Q_{\mathrm{m}}$ and $K_{\mathrm{d}}$ denote adsorption capacity and dissociation constant, respectively.

(3) Adsorption dynamics and elution experiments of MA on two types of adsorbents. Dynamic behaviors of $\beta$-CDPVA-SiO ${ }_{2}$ and $\beta$-CD-SiO ${ }_{2}$ for MA were conducted by the adsorption dynamics and elution experiments. Briefly as follows, $2.0 \mathrm{~g}$ of absorbent was filled into a glass column with $10 \mathrm{~mm}$ of diameter, and formed the bed with $2.5 \mathrm{~mL}$ of volume. Then, the R-MA, S-MA or racemic MA solution with concentration of $0.8 \mathrm{mg} \mathrm{mL}$ was allowed to flow gradually through the column at a rate of three bed volumes per hour $\left(3 \mathrm{BV} \mathrm{h}^{-1}\right)$. Subsequently, the effluents with one bed volume were collected and analyzed to polt the dynamics adsorption curve and calculate the saturated adsorption amounts. Finally, elution experiments were performed by using hydrochloric acid solution $(10 \%, \mathrm{v} / \mathrm{v})$ as eluting agent at the flow rate of $0.5 \mathrm{BV} \mathrm{h}^{-1}$. The eluent was collected and determined to calculate the elution ratio.

\subsection{Adsorptive selectivity of two types of adsorbents}

The selectivity of adsorption to the enantiomers of MA was performed to research the chiral resolution capacity of $\beta$-CD-PVA- $\mathrm{SiO}_{2}$ and $\beta$-CD-SiO${ }_{2}$. In the procedure, $0.5 \mathrm{~g}$ of absorbent was suspended in $100 \mathrm{~mL}$ of racemic MA solution with an initial concentration $\left(C_{0}\right)$ of $1.2 \mathrm{mg} \mathrm{mL}^{-1}$ in the conical flask. Then, the conical flask was stirred by a magnetic stirrer in an incubator at $20^{\circ} \mathrm{C}$. Every $1 \mathrm{~h}, 1 \mathrm{~mL}$ of sample was periodically collected and treated as described in the front. The enantiomers of MA in supernatant were analyzed by HPLC to obtain the concentration of the R-MA and S-MA. In addition, to illustrate the practical value of the materials in chiral drug separation, the concentration of the product after the separation of racemic MA using the method described in experiment part 1.6 were used to evaluate the adsorptive selectivity. The enantiomeric excess (e.e.\%) of the MA solution was calculated by the following equation:

$$
\mathrm{ee} \%=\frac{[\mathrm{S}]-[\mathrm{R}]}{[\mathrm{S}]+[\mathrm{R}]} \times 100 \%,
$$

where $[R]$ and $[S]$ are the concentrations of the R-MA and S-MA in the solution.

\section{Results and discussion}

\subsection{Preparing process and characterization}

(1) FT-IR spectra. The preparing process of tentacle-type adsorbent $\mathrm{CD}-\mathrm{PVA}-\mathrm{SiO}_{2}$ includes three steps as follows (Scheme 1). Firstly, after activated, a great deal of silanol groups reacted with $\gamma$-aminopropyltrimethoxysilane and formed the aminopropyl silica gel $\left(\mathrm{NH}_{2}-\mathrm{SiO}_{2}\right)$. Then, glutaraldehyde was chosen as coupling agent to link the aminopropyl silica and functional macromolecular polyvinyl alcohol (PVA) to form the material with abundant hydroxy groups $\left(\mathrm{PVA}-\mathrm{SiO}_{2}\right)$. Finally, the hydroxy groups and $\beta$ cyclodextrin $(\beta$-CD) were linked by $\gamma$-glycidoxypropyltrimethoxysilane and the tentacle-type material (CD-PVA$\mathrm{SiO}_{2}$ ) was obtained.

From the structure of CD-PVA-SiO ${ }_{2}$ in Scheme 1, it could found that the new tentacle-type adsorbent may provide much more adsorption sites. In order to show the surface structure of the new tentacle-type adsorbent, FT-IR spectra of $\mathrm{SiO}_{2}, \mathrm{PVA}-\mathrm{SiO}_{2}, \beta$-CD-PVA-SiO ${ }_{2}$ were carried out and given in Figure 1. Comparing to the FT-IR spectra of $\mathrm{SiO}_{2}$, the characteristic absorption peak of stretching vibration of C-H bond appeares at $2949 \mathrm{~cm}^{-1}$ (Figure 1(b)), and the characteristic absorption peak of stretching vibration of $\mathrm{C}-\mathrm{O}$ bond are found at $1031 \mathrm{~cm}^{-1}$, respectively. The 


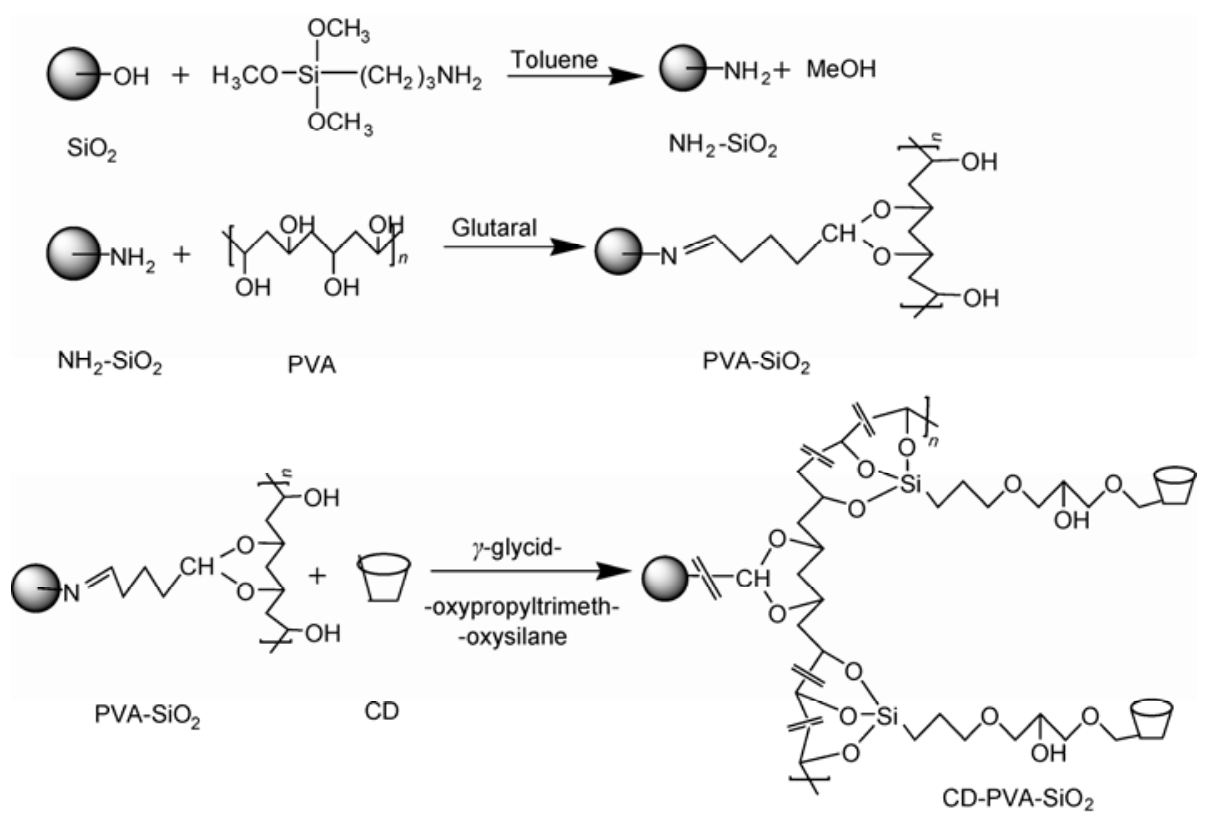

Scheme 1 Synthesis process of composite material CD-PVA-SiO 2 .

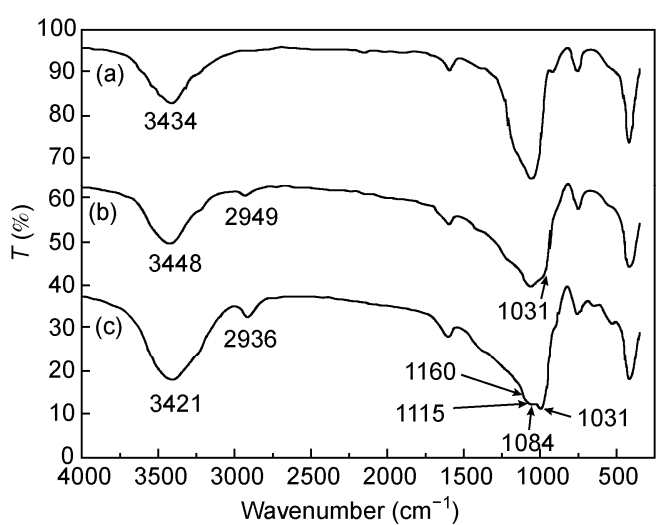

Figure 1 FT-IR spectra of (a) $\mathrm{SiO}_{2}$, (b) PVA-SiO $\mathrm{S}_{2}$ and (c) $\beta$-CD-PVA$\mathrm{SiO}_{2}$.

appearances of these absorption bands show that PVA macromolecules have been grafted onto silica gel surface, and $\mathrm{PVA}-\mathrm{SiO}_{2}$ particles have formed. After the $\mathrm{PVA}-\mathrm{SiO}_{2}$ modified with $\beta$-CD, the FT-IR spectrum was recorded and is given in Figure 1(c). Here, the $\mathrm{O}-\mathrm{H}$ stretching absorption band with the increased intensity is absorbed at $3421 \mathrm{~cm}^{-1}$ and this value shifts about $27 \mathrm{~cm}^{-1}$ to the lower wavenumbers compared to the FT-IR spectrum of PVA-SiO (Figure 1(b)). This result is brought by the short wavenumber of $\mathrm{O}-\mathrm{H}$ stretching absorption band of $\beta$-CD [22]. It shows that $\beta$-CD is modified chemically onto the $\mathrm{PVA}-\mathrm{SiO}_{2}$. Also, the absorption bands at 1160, 1115 and $1084 \mathrm{~cm}^{-1}$ representing the $\mathrm{C}-\mathrm{O}-\mathrm{C}$ bond are other indications to show the chemical modification of the $\beta$-CD onto the PVA-SiO [23].

(2) TGA. The thermogravimetric analysis was performed to determine the amount of $\beta$-CD molecules immo- bilized on $\mathrm{SiO}_{2}$ and PVA-SiO 2 by means of weight balance, and the results are listed in Figure 2. As seen here, there is about $10 \%$ of total weight loss on $\mathrm{PVA}-\mathrm{SiO}_{2}$ by calciantion from 250 to $485^{\circ} \mathrm{C}$. The weight loss can be ascribed to the decomposition of PVA, and is similar to the results reported by other researcher [24,25]. As for samples of $\beta-\mathrm{CD}-\mathrm{SiO}_{2}$ and $\beta$-CD-PVA-SiO $\mathrm{S}_{2}$, the weight loss was determined to be about $13.5 \mathrm{wt} \%$ and $53.1 \mathrm{wt} \%$, respectively. Based on the weight balance, the content of $\beta$-CD on $\mathrm{PVA}_{-} \mathrm{SiO}_{2}$ is estimated to be about $43.1 \mathrm{wt} \%$, three times larger than that of pure $\mathrm{SiO}_{2}$. Obviously, the high content of $\beta$-CD is attributed to the introduction of PVA long chains on silica gel, which provides more reaction sites to increase the amount of $\beta$-CD.

To more exactly elucidate the role of PVA long chain on the ligand density, the contents of $\beta$-CD on both PVAgrafted silica gel and pure silica gel were further measured according to the method described by Dubois [26], and

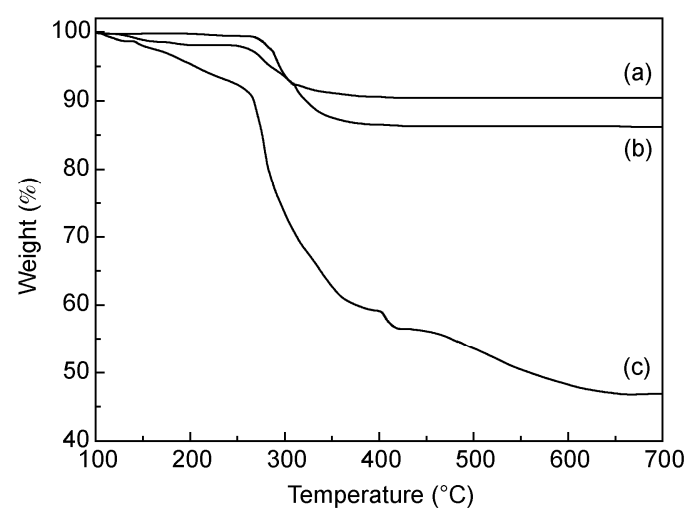

Figure 2 TGA spectrum of (a) $\mathrm{PVA}-\mathrm{SiO}_{2}$, (b) $\beta$-CD-SiO 2 and (c) $\beta$-CDPVA-SiO ${ }_{2}$. 
the results were tabulated in Table 1. It was found that the experimental value of $\beta$-CD on $\mathrm{PVA}-\mathrm{SiO}_{2}$ reached 378 $\mu$ mol $\mathrm{g}^{-1}$, three times larger than pure $\mathrm{SiO}_{2}$. The results matched well with the thermogravimetric analysis.

\subsection{Adsorption studies}

(1) Kinetic adsorption curves of two types of adsorbents. The adsorption kinetics of two types of adsorbents were investigated by using the R-MA and S-MA as model solutes, and shown in Figure 3. It is found that the introduction of PVA on silica gel has significant effect on the adsorption behavior of MA. In case of $\beta$-CD-PVA-SiO ${ }_{2}$, the MA concentration in solution reduced to $0.68 \mathrm{mg} \mathrm{mL}^{-1}$ (R-MA) and $0.77 \mathrm{mg} \mathrm{mL}^{-1}$ (S-MA) after adsorption for $100 \mathrm{~min}$, much lower than those of $\beta$-CD-SiO${ }_{2}$, indicating a higher adsorption rate. The high adsorption kinetics on $\beta$-CD-PVA-SiO ${ }_{2}$ can be ascribed to the increasing amount of $\beta$-CD derived from the PVA on silica gel. Moreover, the curves of kinetic adsorption show that both types of $\beta$-CD adsorbents have better selectivity for R-form of MA instead of its counterpart. This can be explained by the fact that the $\beta$-CD has the chiral binding microenvironment, and can generate more stabile inclusion complex with R-MA than S-MA [27]. This phenomenon has been confirmed by Armstrong [28].

The adsorption of MA on both adsorbents occurred in two stages. For $\beta$-CD-SiO ${ }_{2}$, the first stage underwent MA uptake immediately within $50 \mathrm{~min}$, and then came to the second stage, i.e. the slow adsorption kinetics. Differently, for $\beta$-CD-PVA-SiO ${ }_{2}$, the first stage of solute uptake continued for around $100 \mathrm{~min}$, and then changed to its second stage. The variation in adsorption process is largely influenced by the polymer chains grafted on the adsorbent. Comparing to the non-grafted adsorbent, the $\beta$-CD-PVA-SiO ${ }_{2}$ possesses

Table 1 The supported quantity of $\beta$-CD in two types of adsorbents

\begin{tabular}{ccc}
\hline Samples & $\beta$-CD-SiO & $\beta$-CD-PVA-SiO \\
\hline$\beta$-CD $\left(\mu \mathrm{mol} \mathrm{g}^{-1}\right)$ & $121 \pm 5$ & $378 \pm 7$ \\
\hline
\end{tabular}

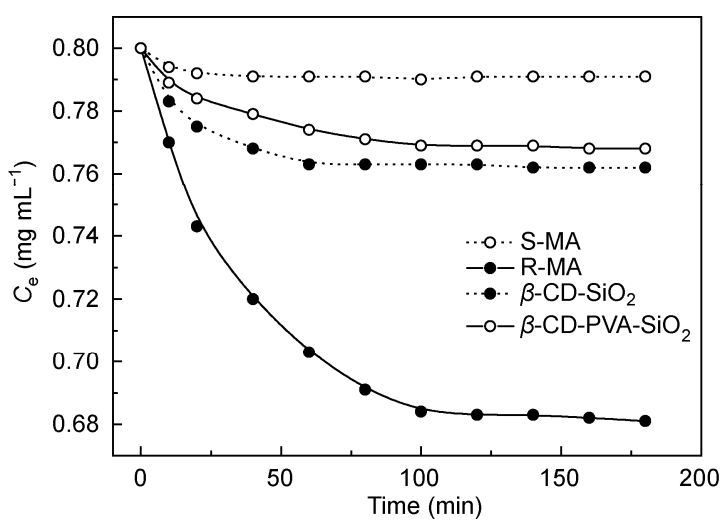

Figure 3 Kinetic adsorption curve for the enantiomers of MA. Temperature: $20^{\circ} \mathrm{C}$; Initial concentration of R-MA and S-MA: $0.8 \mathrm{mg} \mathrm{mL}^{-1}$. more polymer chains anchoring the adsorption sites. It is well known that the film diffusion dominates the solute adsorption during the first adsorption stage. As for adsorbent of $\beta$-CD-PVA-SiO ${ }_{2}$, the polymer long chains on support increase the film diffusion resistance; therefore, the inner adsorption sites on tentacle chains would need more time to contact the MA solutes. So, the first adsorption stage of $\beta$-CD-PVA-SiO ${ }_{2}$ needs longer time period over the nongrafted ones.

To further elucidate the adsorption behavior of tentaclegrafted adsorbent, a series of experiments were carried out to evaluate the influence of temperatures on adsorption kinetics, and the results were shown in Figure 4. It is evident from the results that the adsorption kinetics is dependant upon the temperature. The equilibrium adsorption time reduces with changing the temperature from 20 to $40^{\circ} \mathrm{C}$. It is expected that the increased temperature can help the solutes improve the diffusion mass transfer from the solution to the interior chair ligands.

(2) Adsorption isotherms of the adsorbents. Adsorption isotherms were generated only for the R-MA on $\beta-\mathrm{CD}-\mathrm{SiO}_{2}$ and $\beta$-CD-PVA-SiO ${ }_{2}$ as R-MA could provide better adsorption capacity compared to the S-MA. Effects of solution temperature on R-MA adsorption for the two types of adsorbents at the experimental temperature of 20,30 and $40^{\circ} \mathrm{C}$ were investigated and presented in Figure 5. The figure shows that, the equilibrium adsorption amount decreases with increasing temperature, which indicates that the adsorption is an exothermic process. So the equilibrium adsorption amount for $\beta$-CD-PVA-SiO ${ }_{2}$ containing more chiral ligands reveals more decrease with increasing temperature than $\beta$-CD-SiO${ }_{2}$. Langmuir model fitting the isotherm data to eq. (3) gave the static adsorption capacity $\left(Q_{\mathrm{m}}\right)$ and the dissociation constant $\left(K_{\mathrm{d}}\right)$ (Table 2). The results from Table 2 show that two types of adsorbents have similar dissociation constants at the experimental temperature. It reveals that the introduction of the polymer does not impact the adsorption mechanism. Moreover, the R-MA adsorption capacities based on $\beta$-CD-PVA-SiO ${ }_{2}$ are higher than that of

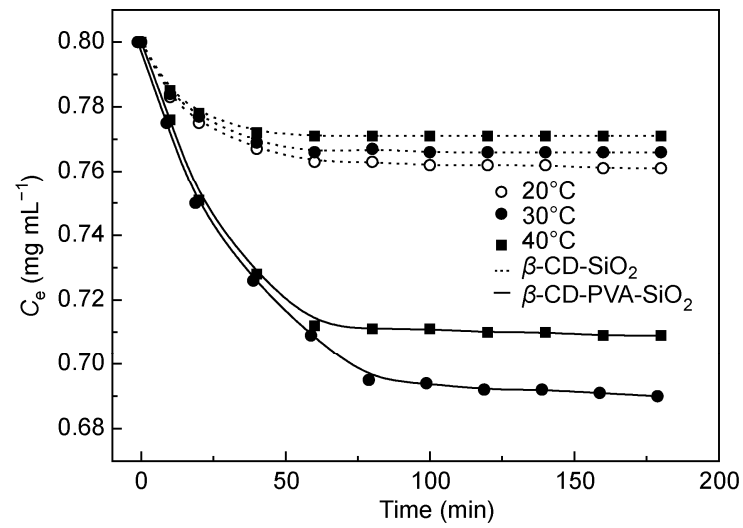

Figure 4 Kinetic adsorption curve for R-MA. Initial concentration of R-MA: $0.8 \mathrm{mg} \mathrm{mL}^{-1}$. 


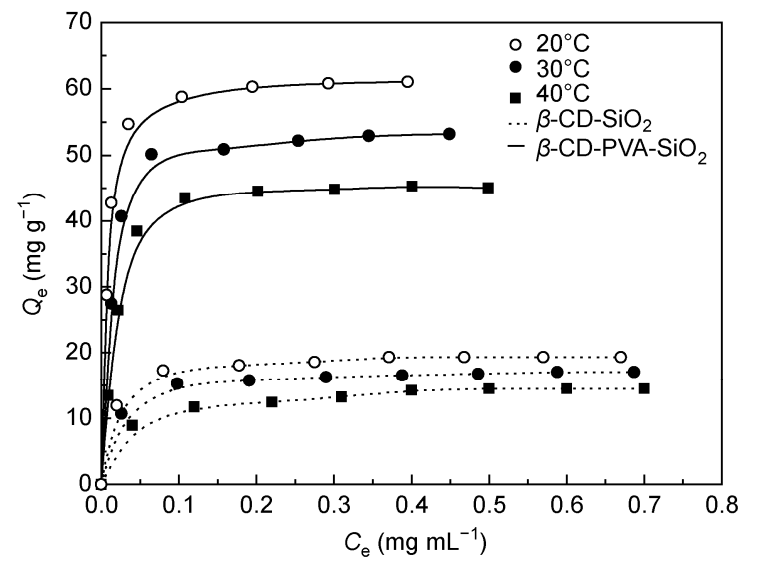

Figure 5 Adsorption isotherms of R-MA at different temperatures.

Table 2 Adsorption properties of the adsorbents for R-MA

\begin{tabular}{llcl}
\hline \multicolumn{1}{c}{ Samples } & & $Q_{\mathrm{m}}\left(\mathrm{mg} \mathrm{g}^{-1}\right)$ & $K_{\mathrm{d}}\left(\mathrm{mg} \mathrm{mL}^{-1}\right)$ \\
\hline$\beta$-CD-SiO & & $19.92 \pm 0.81$ & $0.014 \pm 0.002$ \\
& $30^{\circ} \mathrm{C}$ & $17.54 \pm 0.73$ & $0.017 \pm 0.003$ \\
& $40^{\circ} \mathrm{C}$ & $14.93 \pm 0.62$ & $0.025 \pm 0.003$ \\
$\beta$-CD-PVA-SiO 2 & $20^{\circ} \mathrm{C}$ & $68.97 \pm 2.13$ & $0.014 \pm 0.003$ \\
& $30^{\circ} \mathrm{C}$ & $57.47 \pm 2.01$ & $0.017 \pm 0.002$ \\
& $40^{\circ} \mathrm{C}$ & $52.36 \pm 1.95$ & $0.026 \pm 0.005$ \\
\hline
\end{tabular}

the $\beta-\mathrm{CD}-\mathrm{SiO}_{2}$ at the experimental temperature. As expected, the tentacle-type structure could provide more adsorption sites accessible for chiral molecule approach, thus leading to high static adsorption capacity.

(3) Dynamic adsorption curve. To elucidate clearly the adsorption performance of the adsorbents under actual conditions, breakthrough analysis were performed by using the adsorbents-packed column at $20^{\circ} \mathrm{C}$, and the results were illustrated in Figure 6 and Table 3. It is found that the dynamic binding capacity of R-MA on $\beta$-CD-PVA-SiO ${ }_{2}$ reaches around $46.51 \mathrm{mg} \mathrm{g}^{-1}$, more than three times that on $\beta$-CD-SiO $2\left(15.04 \mathrm{mg} \mathrm{g}^{-1}\right)$. At the same time, the selectivity of adsorption in the dynamic experiment is also rather obvious. For example, the adsorption amount of R-MA(39.43 $\mathrm{mg} \mathrm{g}^{-1}$ ) on $\beta$-CD-PVA-SiO ${ }_{2}$ is far more than that of S-MA $\left(10.53 \mathrm{mg} \mathrm{g}^{-1}\right)$ in the racemic MA solution. These results suggest that the tentacle-type polymer chains have enhanced the multipoint adsorption of the MA and the resolution ability of $\beta$-CD-PVA-SiO ${ }_{2}$ for racemic MA is affirmed. In addition, for these two adsorbents, the dynamic binding capacities of R-MA are lower slightly than their corresponding static adsorption capacities. Analogous phenomenon can be seen for S-MA and racemic MA. It can be explained by the fact that the adsorbent can get full access to the adsorbate in the static adsorption process; differently, in the dynamic tests, the adsorption sites on tentacle-type adsorbent were not sufficiently utilized due to short adsorption time during the breakthrough adsorption [29]. Elution experiment data were tabulated in the Table 3. The results reveal that $\beta$-CDPVA-SiO $\mathrm{S}_{2}$ has the same outstanding elution properties with $\beta$-CD-SiO ${ }_{2}$. It is expected that the excellent retention and elution properties would make the novel tentacle-type adsorbent produce better resolution capacity for chiral drugs.

\subsection{Selectivity of adsorption}

To evaluate the chiral resolution capacity of the adsorbents, the selectivity of adsorption to the enantiomers of MA was carried out on two types of adsorbents and the results were

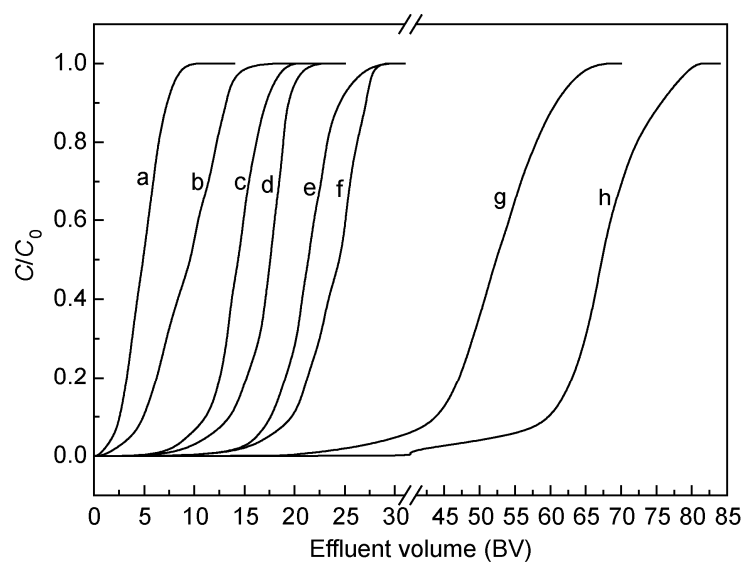

Figure 6 Breakthrough curves of R-MA, S-MA and racemic MA on the column. a, S-MA on $\beta$-CD-SiO ${ }_{2}$; b, S-MA on $\beta$-CD-SiO ${ }_{2}$ in racemic MA solution; c, S-MA on $\beta$-CD-PVA-SiO ${ }_{2}$; d, R-MA on $\beta$-CD-SiO ${ }_{2}$; e, S-MA on $\beta$-CD-PVA-SiO $\mathrm{Sin}_{2}$ racemic MA solution; $\mathrm{f}, \mathrm{R}-\mathrm{MA}$ on $\beta$-CD-SiO $\mathrm{S}_{2}$ in racemic MA solution; g, R-MA on $\beta$-CD-PVA-SiO ${ }_{2}$; h, R-MA on $\beta$-CDPVA-SiO ${ }_{2}$ in racemic MA solution.

Table 3 The results of the dynamic tests

\begin{tabular}{|c|c|c|c|c|}
\hline \multirow{2}{*}{ Samples } & \multicolumn{2}{|c|}{$\beta-\mathrm{CD}-\mathrm{SiO}_{2}$} & \multicolumn{2}{|c|}{$\beta$-CD-PVA-SiO ${ }_{2}$} \\
\hline & R-MA & S-MA & R-MA & S-MA \\
\hline Breakthrough capacity $\left(\mathrm{mg} \mathrm{g}^{-1}\right)$ & $15.04 \pm 0.65$ & $4.52 \pm 0.34$ & $46.51 \pm 1.65$ & $13.53 \pm 0.60$ \\
\hline Desorption ratio $(\%)$ & $97.36 \pm 0.81$ & $98.14 \pm 0.78$ & $96.17 \pm 0.85$ & $96.68 \pm 0.65$ \\
\hline \multirow{2}{*}{ Samples } & \multicolumn{2}{|c|}{$\beta$-CD-SiO 2 (racemic MA) } & \multicolumn{2}{|c|}{$\beta$-CD-PVA-SiO ${ }_{2}($ racemic MA) } \\
\hline & R-MA & S-MA & R-MA & S-MA \\
\hline Desorption ratio $(\%)$ & $97.22 \pm 0.31$ & $99.01 \pm 0.45$ & $96.37 \pm 0.98$ & $96.54 \pm 0.71$ \\
\hline
\end{tabular}


plotted in Figure 7. The figure shows that, with the increase of adsorption time, the change value of ee $\%$ on $\beta$-CD-PVA$\mathrm{SiO}_{2}$ is significantly higher than that on $\beta-\mathrm{CD}-\mathrm{SiO}_{2}$. And ee $\%$ based on $\beta$-CD-PVA- $\mathrm{SiO}_{2}$ reaches about $18.28,3.02$ times than that of $\beta$-CD-SiO ${ }_{2}$ (6.04) after adsorption equilibrium. In addition, Figure 8 shows the ee\% value of racemic MA separated in dynamic condition. It could be found that the time with an ee\% value of $100 \%$ based on $\beta$-CD-PVA-SiO 2 ( $7 \mathrm{~h}$ ) were much longer than that of $\beta$-CD$\mathrm{SiO}_{2}(2 \mathrm{~h})$. What's more, the ee $\%$ value based on $\beta-\mathrm{CD}-\mathrm{SiO}_{2}$ dropped faster than that of $\beta$-CD-PVA-SiO ${ }_{2}$. These certify that the tentacle-type adsorbent is possessed of better selectivity to the enantiomers in racemic solution and more outstanding chiral resolution property over the conventional one. As expected, the novel tentacle-type adsorbent could be as potential CSPs to achieve preparative scale resolution of chiral pharmaceuticals.

\section{Conclusions}

Novel tentacle-type chiral adsorbent and the conventional chiral adsorbent based on $\beta$-CD immobilized on silica were

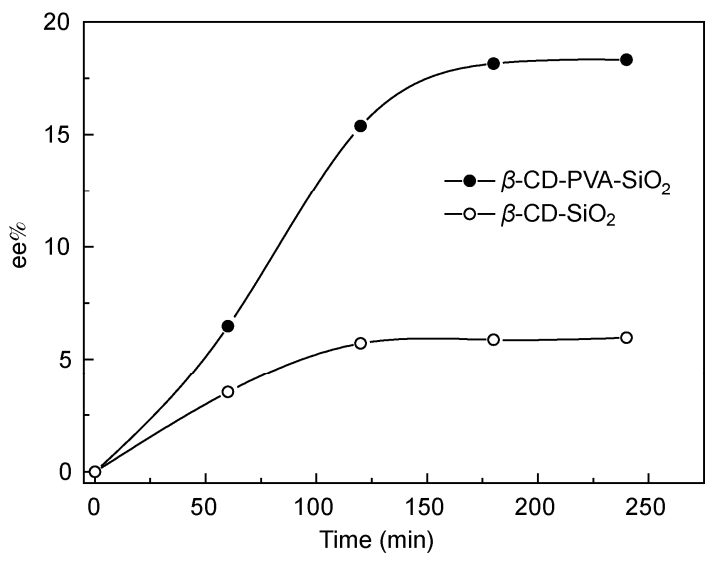

Figure 7 The change of ee\% of racemic MA solution resolution on the presence of the CSPs.

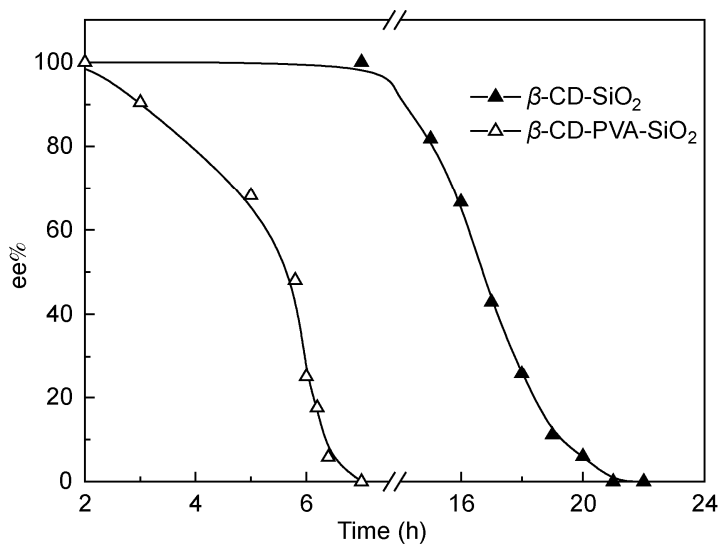

Figure 8 The change of ee\% of racemic MA solution resolution in dynamic condition. synthesized. The results revealed that, owing to the introduction of the PVA, the density of ligands on the novel chiral adsorbent was increased by over $200 \%$ comparing to the conventional one. And then the resolution properties to MA on these two chiral adsorbents were investigated in terms of static, dynamic and selective adsorption. It was found that, the presence of the polymer had no effect on the adsorption mechanism. However, equilibrium adsorption amount to model solute on the tentacle-type chiral adsorbent was increased significantly, over threefold to its un-grafted counterpart; the same outstanding elution property with the conventional chiral adsorbent was exhibited by the novel chiral adsorbent; and the e.e\% value of racemic solution was raised from 6.04 to 18.28 in the static adsorption because of the introdunction of polymer. What's more, the selectivity is still outstanding in dynamic condition. As been seen that, the excellent elution property, lager retention capacity and better selectivity to the enantiomers made the novel tentacle-type chiral adsorbent possess a bright prospects as potential application in preparative scale resolution of chiral pharmaceuticals.

This work was supported by the Doctoral Fund of Ministry of Education of China (20090181110043).

1 Hanna G M, Evans F E. Optimization of enantiomeric separation for quantitative determination of the chiral drug propranolol by ${ }^{1} \mathrm{H}-\mathrm{NMR}$ spectroscopy utilizing a chiral solvating agent. J Pharm Biomed Anal, 2000, 24: 189-196

2 Ali H Y I, Aboul E. Chiral Pollutants, Distributions, Toxicity, and Analysis by Chromatography and Capillary Electrophoresis. Chichester, UK: Wiley, 2004

3 Baley C R, Vaidya V A. Resolution of Racemates by Diastereomeric Salt Formation CR Bayley-Chirality in Industry. New York: John Wiley \& Sons, 1992

4 Pirkle W H, Bowen W E. Preparative separation of enantiomers using hollow-fiber membrane technology. Tetrahedron Asymmetry, 1994, 5: 773-776

5 Goncalves C V, Carpes M J S, Correa C R D, et al. Determination of the kinetic and equilibrium parameters of the $n$-boc-rolipram enantiomers on a chiral stationary phase by high performance liquid chromatography. Chem Eng J, 2007, 133: 151-156

6 Karen L S, De A P, Claudia L, et al. Development of chiral HPLC for selenoamino acids with ICP-MS detection: Application to selenium nutritional supplements. Analyst, 2000, 125: 281-286

7 Gustavsson P E, Larsson P O. Superporous agarose, a new material for chromatography. J Chromatogr A, 1996, 734: 231-240

8 Leonard M. New packing materials for protein chromatography. J Chromatogr B, 1997, 699: 3-27

9 Du K F, Yan M, Wang Q Y, et al. Preparation and characterization of novel macroporous cellulose beads regenerated from ionic liquid for fast chromatography. J Chromatogr A, 2010, 1217: 1298-1304

10 Armstong D W, DeMond W. Cyclodextrin bonded phases for the liquid chromatographic separation of optical, geometrical, and structural isomers. J Chromatogr Sci, 1984, 22: 411-420

11 Shi J H, Ding Z J, Hu Y. Experimental and theoretical studies on the enantioseparation and chiral recognition of mandelate and cyclohexylmandelate on permethylated $\beta$-cyclodextrin chiral stationary phase. Chromatographia, 2011, 74: 319-325

12 Ward T J, Armstrong D W. Improved cyclodextrin chiral phases: A comparison and review. J Liquid Chromatogr, 1986, 9: 407-427 
13 Sharp V S, Letts M N, Risley D S, et al. Enantiomeric separation of dansyl amino acids using macrocyclic antibiotics as chiral mobile phase additives by Narrow-Bore high-performance liquid chromatography. Chirality, 2004, 16: 153-161

14 Martinez R C, Gonzalo E R, Smith N W, et al. Use of a polarembedded stationary phase for the separation of tocopherols by CEC. Electrophoresis, 2006, 27: 4423-4430

15 Sun G Y, Shi Q H, Sun Y. Novel biporous polymeric stationary phase for high-speed protein chromatography. J Chromatogr A, 2004, 1061: 159-160

16 Torres R, Pessela B C, Fuentes M, et al. Supports coated with PEI as a new tool in chromatography. Enzyme Microb Tech, 2006, 39: 711718

17 Wei X, Husson S M. Surface-grafted, molecularly imprinted polymers grown from silica gel for chromatographic separations. Ind Eng Chem Res, 2007, 46: 2117-2124

18 Chanda M, Rempel G L. A new method of gel-coating polyethyleneimine (PEI) on organic resin beads. High capacity and fast kinetics of PEI gel-coated on polystyrene. Ind Eng Chem Res, 2001, 40: 1624-1632

19 Ma Z Y, Guan Y P, Liu X Q, et al. Synthesis of magnetic chelator for high-capacity immobilized metal affinity adsorption of protein by cerium initiated graft polymerization. Langmuir, 2005, 21: 6987-6994

20 Bajpal A K, Saini R. Preparation and characterization of novel biocompatible cryogels of poly(vinyl alcohol) and egg-albumin and their water sorption study. Mater Sci, 2006, 17: 49-61

21 Goswami A, Singh A K. 1,8-Dihydroxyanthraquinone anchored on silica gel: Synthesis and application as solid phase extractant for lead
(II), zinc (II) and cadmium (II) prior to their determination by flame atomic absorption spectrometry. Talanta, 2002, 58: 669-678

22 Abay I, Denizli A, Biskin E, et al. Removal and pre-concentration of phenolic species onto b-cyclodextrin modified poly(hydroxyethylmethacrylateethyleneglycoldimethacrylate) microbeads. Chemosphere, 2005, 61: 1263-1272

23 Liu Y Y, Fan X D. Synthesis, properties and controlled release behaviors of hydrogel networks using cyclodextrin as pendant groups. Biomaterials, 2005, 26: 6367-6374

24 Krissanasaeranee M, Vongsetskul T, Rangkupan R, et al. Preparation of ultra-fine silica fibers using electrospun poly(vinyl alcohol)/ silatrane composite fibers as precursor. J Am Ceram Soc, 2008, 91: 2830-2835

25 Nakane K, Yamashita T, Iwakura K, et al. Properties and structure of poly(vinyl alcohol)/silica composites. J Appl Polym Sci, 1999, 74: 133-138

26 Dubois M, Gilles K A, Hamilion J K, et al. Colorimetric method for determination of sugars and related substances. Anal Chem, 1956, 28 : 350-359

27 Salvatierra D, Sánchez-Ruiz X, Garduňo R, et al. enantiodifferentiation by complexation with [beta]-cyclodextrin: Experimental (NMR) and theoretical (MD, FEP) studies. Tetrahedron, 2000, 56: 3035-3041

28 Armstrong D W, Han Y I, Han S M. Liquid chromatographic resolution of enantiomers containing single aromatic rings with $\beta$-cyclodextrinbonded phases. Anal Chim Acta, 1988, 208: 275-281

29 Amarasinghe B M, Williams R A. Tea waste as a low cost adsorbent for the removal of $\mathrm{Cu}$ and $\mathrm{Pb}$ from wastewater. Chem Eng J, 2007, 132: 299-309

Open Access This article is distributed under the terms of the Creative Commons Attribution License which permits any use, distribution, and reproduction in any medium, provided the original author(s) and source are credited. 